\title{
A Spiking Neural P System Simulator Based on CUDA
}

\author{
Francis George C. Cabarle ${ }^{1}$, Henry Adorna ${ }^{1}$, \\ and Miguel A. Martínez-del-Amor ${ }^{2}$ \\ 1 Algorithms \& Complexity Lab, \\ Department of Computer Science, \\ University of the Philippines Diliman, \\ Diliman 1101 Quezon City, Philippines \\ fccabarle@up.edu.ph, hnadorna@dcs.upd.edu.ph \\ 2 Research Group on Natural Computing, \\ Department of Computer Science and Artificial Intelligence, \\ University of Seville, \\ Avda. Reina Mercedes s/n, 41012 Sevilla, Spain \\ mdelamor@us.es
}

\begin{abstract}
In this paper we present a Spiking Neural P system (SNP system) simulator based on graphics processing units (GPUs). In particular we implement the simulator using NVIDIA CUDA enabled GPUs. The massively parallel architecture of current GPUs is very suitable for the maximally parallel computations of SNP systems. We simulate a wider variety of SNP systems, after presenting a previous work on SNP system matrix representation which led to their simulation in GPUs, and the simulation algorithm included here. Finally, we compare and present the performance speedups of the CPU-GPU based simulator over the CPU only simulator.
\end{abstract}

Keywords: Membrane Computing, Spiking Neural P systems, Parallel Computing, GPU Computing, CUDA.

\section{Introduction}

Inspiration taken from nature can help us define new computing models, with the aim of providing efficient solutions to the limitations of conventional models of computation. In this respect Membrane Computing, a research area initiated by Gheorghe Păun in 1998 [14, provides distributed, parallel, and nondeterministic computing models known as $P$ systems. These models are basically abstractions of the compartmentalized structure and parallel processing of biochemical information in biological cells.

Many $\mathrm{P}$ system variants have been defined in literature, and many of them have been proven to be computationally complete. Moreover, several general classifications of $\mathrm{P}$ systems are considered depending on the level of abstraction: cell-like (a rooted tree where the skin or outermost cell membrane is the root, 
and its inner membranes are the children or leaf nodes in the tree), tissue-like (a graph connecting the cell membranes) and neural-like (a directed graph, inspired by neurons interconnected by their axons and synapses). The last type refers to Spiking Neural P systems (in short, SNP systems), where the time difference (when neurons fire and/or spike) plays an essential role in the computations [11.

One key reason of interest for $\mathrm{P}$ systems is that they are able to solve computationally hard problems (e.g. NP-complete problems) usually in polynomial to linear time only, but requiring exponential space as trade off. These solutions are inspired by the capability of cells to produce an exponential number of new membranes via methods like mitosis (membrane division) or autopoiesis (membrane creation). However, because of this massively parallel, distributed and nondeterministic nature of $\mathrm{P}$ systems, they are yet to be fully implemented in vivo, in vitro, or even in silico. Thus, practical computations of $\mathrm{P}$ systems are driven by silicon-based simulators.

Since $\mathrm{P}$ systems were introduced, many simulators have been produced by using different software and hardware technologies [7. In practice, $\mathrm{P}$ system simulations are limited by the physical laws of silicon architectures, which are often inefficient or not suitable when dealing with $\mathrm{P}$ system features, such as the exponential workspace creation and massive parallelism. However, in order to improve the efficiency of the simulators, it is necessary to exploit current technologies, leading to solutions in the area of High Performance Computing. In this way, many simulators have been developed over highly parallel platforms, including reconfigurable hardware as in FPGAs [15], CPU-based clusters [6], as well as the Graphical Processing Units (GPUs) [34]. These efforts show that parallel devices are very suitable in simulating $\mathrm{P}$ systems, at least for the first few $\mathrm{P}$ system variants to have been introduced (transition and active membrane $\mathrm{P}$ systems). Efficiently simulating SNP systems would thus require new attempts in parallel computing.

GPUs are the leading exemplars of modern high throughput-oriented architectures [9]. Nowadays, these kinds of processors are used for tackling problems where parallelism is prevalent. Indeed, GPUs have been successfully used to speedup many parallel applications. Modern GPUs are not limited only to graphics processing, as the first graphic cards were, since current GPUs can now be used for general purpose computations [10; they are now multi-core and data-parallel processors [12]. By using GPU computing, also known as GPGPU (General Purpose computing on the GPU), a programmer can achieve with a single GPU, a throughput similar to that of a CPU based cluster [1017]. Thus, the main advantages of using GPUs are their low-cost, low-maintenance and low power consumption relative to conventional parallel clusters and setups, while providing comparable or improved computational power. Moreover, parallel computing concepts such as hardware abstraction, scaling, and so on are handled efficiently by current GPUs.

Given that SNP systems have already been represented as matrices due to their graph-like properties [16], simulating them in parallel devices such as GPUs 
is the next natural step. Matrix algorithms are well known in parallel computing literature, including GPUs [8], due to the highly parallelizable nature of linear algebra computations mapping directly to the data-parallel GPU architecture.

Previously, SNP systems have been faithfully simulated in GPUs using their matrix representation [1. This simulator combined both the object oriented programming language (OOPL) Python (CPU part) and CUDA/C (GPU part) codes, and so it has been improved [2], in performance, by using the PyCUDA [13] library. In this paper we present an extension of [2] in order to simulate SNP systems with more general regular expressions associated to their firing rules. This extension allows us to simulate larger and wider varieties of SNP systems in order to test the speedup achieved by the GPU and CPU based simulators.

This paper is organized as follows: Section 2 introduces SNP systems formally, as well as their matrix representation. Section 3 provides background for GPU computing with CUDA. The design of the simulator and simulation results are given in Section 4 and Section [5] respectively. Finally, conclusions, future work, acknowledgements, and references end this paper.

\section{Spiking Neural P Systems}

In this section the SNP system model is introduced, together with a matrix representation of the model. This representation is the basis for the simulation algorithm in this paper. Additionally, the two examples used to test and analyse the simulator are also described.

\subsection{The SNP System Model}

Many variants of SNP systems have been introduced in recent works, such as those with delays, weights, extended firing rules, deterministic systems, division, budding, and so on. Each one has specific features in complexity, but the majority of them have been shown to be computationally complete [115. This paper is focused on a restricted variant of SNP systems, with no delays associated to the rules (i.e. neurons fire immediately once they are able to do so), which are of the following form:

Definition 1. An SNP system without delay, of degree $m \geq 1$, is a construct of the form

$$
\Pi=\left(O, \sigma_{1}, \ldots, \sigma_{m}, \text { syn }, \text { in }, \text { out }\right)
$$

where:

1. $O=\{a\}$ is the alphabet made up of only one object a, called spike;

2. $\sigma_{1}, \ldots, \sigma_{m}$ are $m$ neurons of the form

$$
\sigma_{i}=\left(n_{i}, R_{i}\right), 1 \leq i \leq m,
$$

where: 
(a) $n_{i} \geq 0$ gives the initial number of spikes (a) contained in neuron $\sigma_{i}$;

(b) $R_{i}$ is a finite set of rules of the following forms:

(b-1) $E / a^{c} \rightarrow a^{p}$, are known as Spiking rules, where $E$ is a regular expression over $a$, and $c \geq 1$, for $p \geq 1$ number of spikes are produced (with the restriction $c \geq p$ ), transmitted to each adjacent neuron with $\sigma_{i}$ as the originating neuron, and $a^{c} \in L(E)$;

(b-2) $a^{s} \rightarrow \lambda$, are known as Forgetting rules, for $s \geq 1$, such that for each rule $E / a^{c} \rightarrow a^{p}$ of type (b-1) from $R_{i}, a^{s} \notin L(E)$;

3. syn $=\{(i, j) \mid 1 \leq i, j \leq m, i \neq j\}$ are the synapses i.e. connections between neurons;

4. in, out $\in\{1,2, \ldots, m\}$ are the input and output neurons, respectively.

A spiking rule (type (b-1)) $r_{s} \in R_{i}$, where $1 \leq i \leq m$, is applied if the corresponding $\sigma_{i}$ contains $k$ spikes, $a^{k} \in L(E)$ and $k \geq c$. This means that consuming (removing) $c$ spikes (thus only $k-c$ spikes remain in $\sigma_{i}$ ), the neuron is fired, producing $p$ spikes that reach all $\sigma_{j}$ neurons immediately (no delays) such that $(i, j) \in$ syn. An SNP system whose spiking rules have $p=1$ (they produce only one spike) is said to be of the standard type (non-extended). Forgetting rules (type (b-2)) are selected if $\sigma_{i}$ contains $s$ spikes. Thus, $s$ spikes are 'forgotten' or removed from the neuron once the rule is applied. Finally, a special case of (b-1) are rules of type (b-3) where $a^{c} \rightarrow a, L(E)=\left\{a^{c}\right\}, k=c, p=1$.

It is noteworthy that the neurons in an SNP system operate in parallel and in unison, under a global clock [1]. Similar to the usual way of using rules in other $\mathrm{P}$ system variants, there is maximal parallelism at the level of the system, in the sense that in each step all neurons which can spike (i.e. fire a rule) have to do it. However, only one rule can be applied at a given time in each neuron [1116. The nondeterminism of SNP systems comes with this fact: $L(E) \cap L\left(E^{\prime}\right) \neq \emptyset$ for two different spiking rules with regular expressions $E$ and $E^{\prime}$, i.e. exactly one rule among several other applicable rules is chosen nondeterministically.

\subsection{Examples of SNP Systems}

Next, two specific SNP systems are described to show their formal description based on Definition 1. The examples are from [16] and 11], and they are called $\Pi_{1}$ and $\Pi_{2}$ respectively. These examples are used in Section 5 to analyse the performance of the simulator.

The SNP system $\Pi_{1}$ shown in Figure 1 generates all numbers in the set $\mathbb{N}$ $\{1\}$ (so it doesn't halt) and the outputs of the computation are derived from the time difference between the first spike of the output neuron (to the environment) and its succeeding spikes. It can be seen that a total system ordering is given to neurons (from $\sigma_{1}$ to $\sigma_{3}$ ) and rules (from $R_{1}$ to $R_{5}$ ). This SNP system is of the form $\Pi_{1}=\left(\{a\}, \sigma_{1}, \sigma_{2}, \sigma_{3}\right.$, syn, out $)$ where the first neuron $\sigma_{1}=\left(2,\left\{R_{1}, R_{2}\right\}\right)$, $n_{1}=2, R_{1}=\left\{a^{2} / a \rightarrow a\right\}, R_{2}=\left\{a^{2} \rightarrow a\right\},\left(\sigma_{2}\right.$ to $\sigma_{3}$ and their $n_{i}$ s and $R_{i}$ s can be similarly shown $)$, syn $=\{(1,2),(1,3),(2,1),(2,3)\}$ are the synapses for $\Pi_{1}$, and the output neuron out $=\sigma_{3}$, as can be seen by the arrow not pointing to any neuron. $\Pi_{1}$ has no input neuron. 


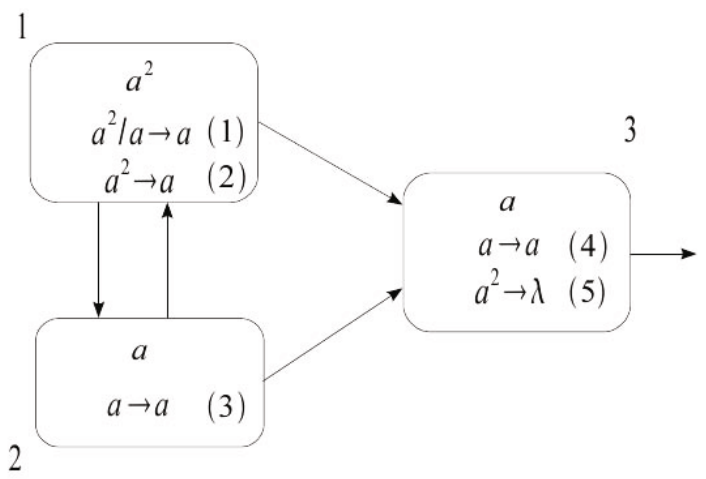

Fig. 1. $\Pi_{1}$, an SNP system generating all numbers in the set $\mathbb{N}-\{1\}$, from [16].

A second SNP system $\Pi_{2}$, adapted from Figure 8 of [11, is shown in Figure [2. $\Pi_{2}$ is larger (in terms of the number of neurons and rules) than $\Pi_{1}$ (from Figure 10 and is formally defined as follows:

$$
\Pi_{2}=\left(\{a\}, \sigma_{1}, \sigma_{f_{1}}, \sigma_{f_{2}}, \sigma_{f_{3}}, \sigma_{f_{4}}, \sigma_{f_{5}}, \sigma_{f_{6}}, \sigma_{l_{h}}, \sigma_{\text {out }}, \text { syn, out }\right)
$$

where the $\sigma$ (neuron) labelling are taken from [1]. Including the total ordering of the rules in $\Pi_{2}$, we have the following:

$\sigma_{1}=\left(0,\left\{R_{1}, R_{2}\right\}\right), n_{1}=0$ ( $\sigma_{1}$ in this case initially has no spikes), $R_{1}=$ $a^{3}(a a)^{+} / a^{2} \rightarrow a, R_{2}=a^{3} \rightarrow a$,

$\sigma_{f_{1}}=\left(0,\left\{R_{3}\right\}\right), n_{f_{1}}=0, R_{3}=\{a \rightarrow a\}$,

$\sigma_{f_{2}}=\left(0,\left\{R_{4}\right\}\right), n_{f_{2}}=0, R_{4}=\{a \rightarrow a\}$,

$\sigma_{f_{3}}=\left(0,\left\{R_{5}, R_{6}\right\}\right), n_{f_{3}}=0, R_{5}=\left\{a^{2} \rightarrow a\right\}, R_{6}=\{a \rightarrow \lambda\}$,

$\sigma_{f_{4}}=\left(0,\left\{R_{7}, R_{8}\right\}\right), n_{f_{4}}=0, R_{7}=\left\{a^{2} \rightarrow a\right\}, R_{8}=\{a \rightarrow \lambda\}$,

$\sigma_{f_{5}}=\left(0,\left\{R_{9}\right\}\right), n_{f_{5}}=0, R_{9}=\{a \rightarrow a\}$,

$\sigma_{f_{6}}=\left(0,\left\{R_{10}, R_{11}\right\}\right), n_{f_{6}}=0, R_{10}=\left\{a^{3} \rightarrow \lambda\right\}, R_{11}=\left\{a^{2} \rightarrow 2\right\}$,

$\sigma_{l_{h}}=\left(0,\left\{R_{12}, R_{13}\right\}\right), n_{l_{h}}=0, R_{12}=\left\{a^{2} \rightarrow a\right\}, R_{13}=\{a \rightarrow \lambda\}$,

$\sigma_{\text {out }}=\left(0,\left\{R_{14}\right\}\right), n_{\text {out }}=0, R_{14}=\{a \rightarrow a\}$

As with $\Pi_{1}$, it is evident that for $\Pi_{2}$ we have 17 synapses connecting the neurons $(\sigma \mathrm{s}) . \Pi_{2}$ doesn't have an input $\sigma$ but it has out $=\sigma_{\text {out }}$.

\subsection{Matrix Representation of SNP Systems}

In [16], a matrix representation of SNP systems without delays was introduced. By using this algebraic representation, it is easy to describe a simulation algorithm for computing configurations in SNP systems without delays by only using vector-matrix operations. The matrix representation inherently and conveniently involves another SNP system variant, the variant with extended firing rules where neurons can fire more than one spike at a given time [5]. Another convenient use for this algebraic representation is the possibility of 
performing backwards computation, from the current configuration to an earlier configuration. This representation makes use of the following vectors and matrix definitions:

Configuration vector $C_{k}$ is the vector containing all spikes in every neuron on the $k$ th computation step/time, where $C_{0}$ is the initial vector containing all spikes in the system at the beginning of the computation. For $\Pi_{1}$ (the example in Figure 1) $C_{0}=<2,1,1>$. For $\Pi_{2}$ we don't have a default $C_{0}$ so we will assign several later, for the purpose of simulation. For this work, since we are not simulating SNP systems with delays, the steps focused on are purely computational and not time steps.

Spiking vector $S_{k}$ shows, at a given configuration $C_{k}$, if a rule is applicable (having value 1 ) or not (having value 0 instead). For $\Pi_{1}$ we have a spiking vector $S_{k}=<1,0,1,1,0>$ given $C_{0}$. Note that a second spiking vector, $S_{k}^{\prime}=<$ $0,1,1,1,0>$, is possible and valid if we use rule (2) over rule (1) instead (but not both at the same time). Validity in this case means that only one among several applicable rules is used and thus represented in the spiking vector. We recall the applicability of rules from the definitions of (b-1) and (b-3). It is worth mentioning that there are several $S_{k}$ s for every possible rule selection in the system. We cannot have an $S_{k}^{\prime \prime}=<1,1,1,1,0>$ because we cannot use rule (1) and rule (2) at the same time, hence this $S_{k}$ is invalid.

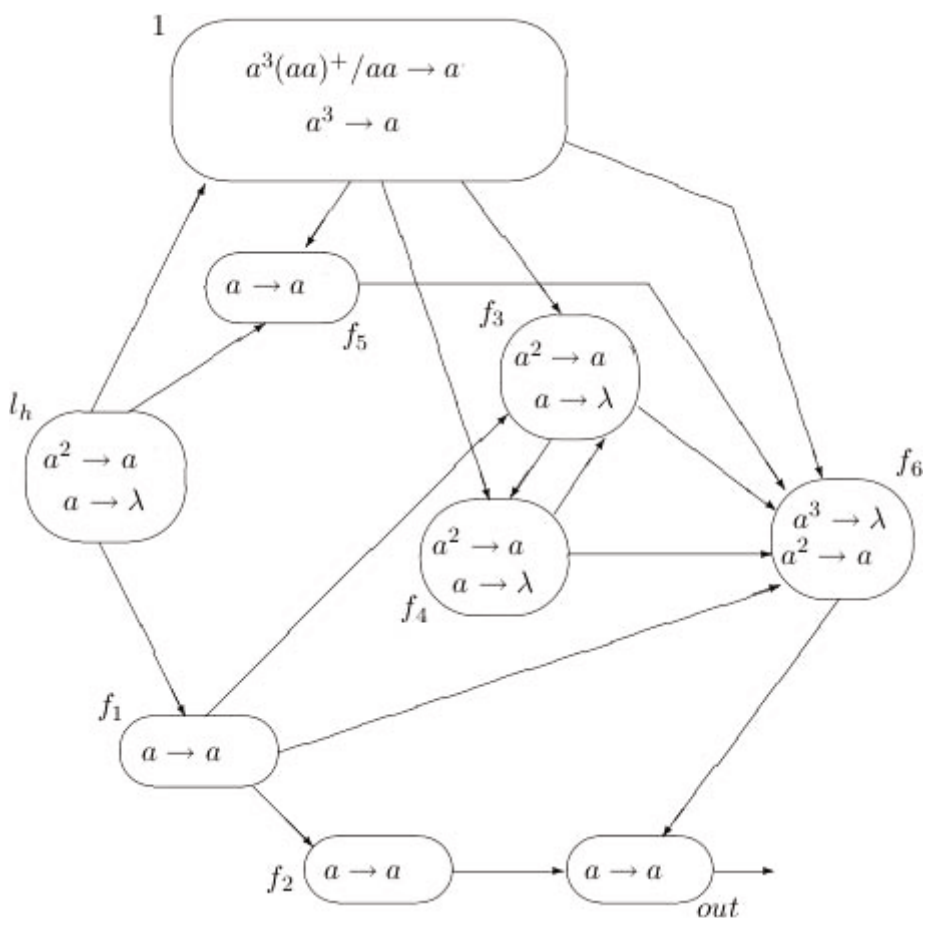

Fig. 2. $\Pi_{2}$, a larger SNP system with 9 neurons and 14 rules, adapted from 11 
Spiking transition matrix $M_{S N P}$ is a matrix comprised of $a_{i j}$ elements where $a_{i j}$ is given as

\section{Definition 2}

$$
a_{i j}=\left\{\begin{array}{r}
-c, \text { rule } r_{i} \text { is in } \sigma_{j} \text { and is applied consuming c spikes; } \\
p, \text { rule } r_{i} \text { is in } \sigma_{s}(s \neq j \text { and }(s, j) \in \text { syn }) \\
\text { and is applied producing } p \text { spikes in total; } \\
0, \text { rule } r_{i} \text { is in } \sigma_{s}(s \neq j \text { and }(s, j) \notin \text { syn }) .
\end{array}\right.
$$

In such a scheme, rows represent rules and columns represent neurons. As mentioned earlier, SNP systems with extended firing rules can be also represented by the spiking transition matrix.

A negative entry in the spiking transition matrix corresponds to the consumption of spikes. Thus, it is easy to observe that each row has exactly one negative entry, and each column has at least one negative entry [16]. For $\Pi_{1}$ and $\Pi_{2}$, the spiking transition matrices $M_{\Pi_{1}}$ and $M_{\Pi_{2}}$ are shown in the equations (1) and (2), respectively.

$$
M_{\Pi_{1}}=\left(\begin{array}{cccc}
-1 & 1 & 1 \\
-2 & 1 & 1 \\
1 & -1 & 1 \\
0 & 0 & -1 \\
0 & 0 & -2
\end{array}\right)
$$

Finally, the following equation provides the configuration vector at the $(k+1)$ th step, given the configuration vector $\left(C_{k}\right)$ and spiking vector $\left(S_{k}\right)$ at the $k t h$ step, and $M_{\Pi}$ :

$$
C_{k+1}=C_{k}+S_{k} \cdot M_{\Pi}
$$




\section{GPU Computing}

High Performance Computing provides solutions for improving the performance of software applications by using accelerators or many-core processors. In this respect, Graphics Processing Units (GPUs) have been consolidated as accelerators thanks to their throughput-oriented highly-parallel architecture [9, as well as their low-power consumption and low-cost compared to other parallel clusters and setups. At the moment, for around \$500, the latest GPUs of NVIDIA with 512 cores and with a performance comparable to a cluster of multi-core CPUs, is readily available at consumer electronics stores. The programming and architectural aspects of GPUs are described in this section.

\subsection{Compute Unified Device Architecture (CUDA)}

As many-core based platforms, GPUs are massively parallel processors which have high chip scalability in terms of processing units (core), and high bandwidth with internal GPU memories. The common CPU architectures are composed of transistors associated with different computing tasks: control, caching, DRAM, and ALU (arithmetic and logic). In contrast, only a fraction of the CPU's transistors allocated for control and caching are used by GPUs, since far more transistors are devoted for ALU [12. This architectural difference is a very distinct and significant reason why GPUs offer larger performance increase over CPU only implementation of parallel code working on large amounts of input data.

The programmability of GPUs has been focused on graphics, but using a type of parallel computing technique called GPGPU (General Purpose computation on GPUs), the large amount of internal cores can be used in parallel for accelerating the execution of data-parallel algorithms. In order to provide a straightforward, easy-to-learn, and scalable programming framework for GPUs, NVIDIA corporation (a well known manufacturer of graphics processors) introduced the Compute Unified Device Architecture (CUDA) in 2007 [12. CUDA is a programming model and hardware architecture for general purpose computations in NVIDIA's GPUs (G80 and newer family of GPUs) 12. By extending popular languages such as $C$, CUDA allows programmers to easily create software that will be executed in parallel, avoiding low-level graphics and hardware primitives [17]. Among the other benefits of CUDA are abstracted and automated scaling: GPUs with more cores will make the parallelized code run faster than GPUs with fewer cores [17].

As seen in Figure 3 CUDA implements a heterogeneous computing architecture, where two different parts are often considered: the host (CPU side) and the device (GPU side). The host/CPU part of the code is responsible for controlling the program execution flow, allocating memory in the host or device/GPU, and obtaining results from the device by executing specific codes. The device (or devices if there are several GPUs in the setup) acts as a parallel co-processor to the host. The host outsources the parallel part of the program as well as the data to the device, since it is more suited to parallel computations than the host. 


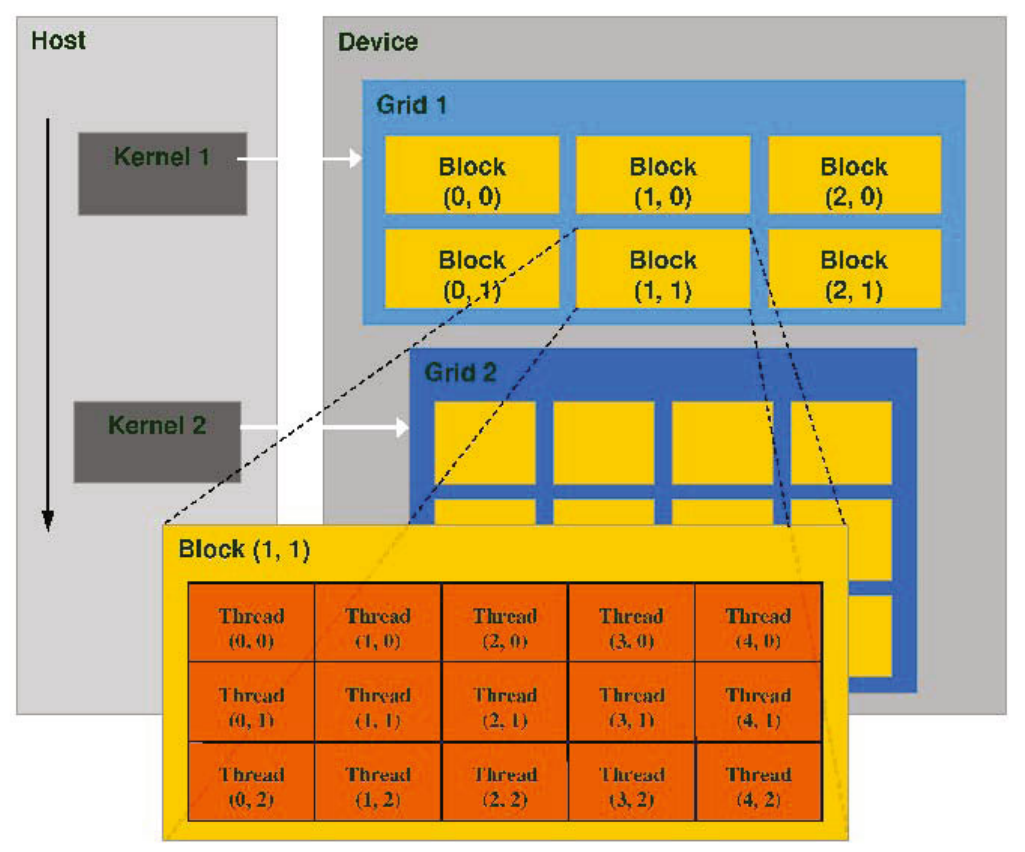

Fig. 3. Structure of the CUDA programming model, from [4]

The code to be executed in a GPU is written in CUDA C (CUDA extended ANSI C programming language). The parallel distribution of the execution units (threads) in CUDA can be split up into multiple threads within multiple thread blocks, each contained within a grid of (thread) blocks (see Figure 3). These grids belong to a single device/single GPU. Each device has multiple cores, each capable of running its own block of threads [1217]. A function known as a kernel function is one that is called from the host but executed in the device. Using kernel functions, the programmer can specify the GPU resources: the layout of the threads (from one to three dimensions) and the blocks (from one to two dimensions). GPUs with the same architecture as the one used in this work has a maximum number of threads per block equal to 512. The maximum size of each dimension of a thread block is $(512 \times 512 \times 64)$, pertaining to the $x, y$, and $z$ dimensions of a block respectively. Lastly, the maximum size of each dimension of a grid of thread block is $(65535 \times 65535 \times 1)$ for the grid's $x, y$, and $z$ dimensions.

\subsection{SNP System GPU Simulation Considerations}

To successfully simulate SNP systems, the input files are file versions of $C_{k}, S_{k}$, $M_{S N P}$, and a file $r$ containing the list of rules $R_{i}$. Since SNP systems involve 
regular expressions (similar to other $\mathrm{P}$ system variants), string manipulation of the number of spikes in a neuron satisfying the regular expression $E$ is involved. An OOPL such as Python is very well suited for string manipulation. For the computations involving linear algebra, since $C_{k}, S_{k}$ and $M_{S N P}$ only have integral values, the $\mathrm{C}$ programming language (which NVIDIA extended for their purposes as CUDA C) is well suited. Another reason for using $\mathrm{C}$ is because of this $\mathrm{C}$ language extension of CUDA. In actuality, only the kernel functions are written in $\mathrm{C}$, and those functions are embedded within the Python code. A row-major ordering, a well known linear array input format where rows are placed one after another from top to bottom, was used to input the elements of $M_{S N P}$. As an example, row-major ordering of $M_{\Pi_{1}}$ is: $1,1,1,2,1,1,1,1,1,0,0,1,0,0,2$.

The current dichotomy of the CUDA programming model is that the data or inputs are loaded and 'prepared' in the host part, then they are moved to the device part and the parallel part of the process is executed on the data. Finally, the results of the parallel processing are moved back to the host for further processing. As seen from Figure 3 kernel functions are called from the host sequentially but once executed in the device, their blocks and the threads within these blocks are executed in parallel.

PyCUDA was chosen in order to fully utilize the speedup of CUDA as well as minimize development time, and is a Python programming interface to CUDA 13. PyCUDA was developed by mathematician Andreas Klöckner for his dissertation for a more efficient parallel computing on CUDA using Python: safer in terms of memory handling, object cleanup (among others), and faster in terms of development time via abstractions [13].

\section{Simulating SNP Systems in CUDA}

In this paper we designate as snpgpu-sim3 our improved simulator, as compared to snpgpu-sim2 from [2]. The simulation algorithm for snpgpu-sim3, as with snpgpu-sim2, is shown in Algorithm 1] Algorithm 1 shows which part of the algorithm (and hence the simulation) is run: whether in the host part or the device part, or both.

Firstly, the simulation inputs are loaded as mentioned in Subsection [3.2, as white space separated files (for $C_{k}, S_{k}$, and $M$ ) and using delimiters '@' and ' $\&$ ' in $r$ to delineate one rule from another in the same neuron, and from one neuron to another, respectively. For example, the file $r$ pertaining to the rules of $\Pi_{1}$ contains: aa 11 @aa 2 186a116a $11 @ a a 10 . R_{1}$ of $\Pi_{1}$ pertains to $a a$ 1 1. In this encoding, the first part is the regular expression $E$ (in this case, $a^{2}$ or $a a$ ), the middle part is the number of spikes consumed (one spike) and lastly, the number of spikes produced (again, one spike).

In part II, the number of spikes in a neuron are checked if they satisfy the regular expression $E$. In snpgpu-sim2 only rules of the form (b-3), and not (b-1), were simulated, thus snpgpu-sim3 can simulate more general SNP systems. A function created in Python, chkRegExp ( regexp, spikNum) returns a boolean value of True if and only if the number of spikes given by spikNum (and hence 
the number of spikes in a $\sigma$ ) are in the language generated by the regular expression regexp. Otherwise, function chkRegExp returns a boolean False. Part II is responsible for generating all possible and valid $S_{k} \mathrm{~s}$ out of the current $C_{k} \mathrm{~s}$ and the rule file $r$. Note that not all possible $S_{k}$ s (strings of $1 \mathrm{~s}$ and $0 \mathrm{~s}$ ) are valid, since exactly one rule only, chosen nondeterministically, is applied per neuron per time step. The simulation 'implements' the nondeterminism (as nondeterminism is yet to be fully realized in hardware) by producing all the possible and valid $S_{k}$ s for the given $C_{k}$ s, and proceeds to compute each of the $C_{k+1}$ from these.

The process by which all possible and valid $S_{k}$ s are produced is as follows: Once all the rules in the system are identified, given the current $n_{i}$ s (number of spikes present in each of the $\sigma_{i} \mathrm{~s}$ ), the $\{1,0\}$ strings (at the moment they are treated as strings, and then as integral values later on) are produced on a per neuron level. As an example, given that $n_{1}=2$ for $\Pi_{1}$, and its two rules $R_{1}$ and $R_{2}$, we have the neuron-level strings '10' (we choose to use $R_{1}$ instead of $R_{2}$ ) and '01' (use $R_{2}$ instead of $R_{1}$ ). For $\sigma_{2}$ we only have '1' ( $R_{3}$ of $\sigma_{2}$ has the needed single spike, and it has only one rule) while $\sigma_{3}$ gives us ' 10 ' since its single spike enables $R_{4}$ only and not $R_{5}$. After producing the neuron-level $\{1,0\}$ strings, the strings are exhaustively paired up, from left to right (since there is a need for ordering), until finally all the valid and possible $S_{k}$ s from the current $C_{k} \mathrm{~s}$ are produced. For $\Pi_{1}$, given $C_{0}=<2,1,1>$ we have $S_{k} \mathrm{~s}(1,0,1,1,0)$ and $(0,1,1,1,0)$.

Part III performs Equation [3, which is done in parallel in the device. The previously loaded values of $C_{k}, S_{k}, r$, and $M$ which were treated as strings (for the purposes of concatenation, regular expression checking, among others) are now treated as integral values. Each thread in the device contains either a matrix element from $M$ or a vector element from $S_{k}$ or $C_{k}$, and Equation 3 is performed in parallel. The newly produced $C_{k+1}$ are then moved back from the device to the host. Part IV then checks whether to proceed or to stop based on 2 stopping criteria for the simulation: (I) if a zero vector (vector of zeros) is encountered, (II) if the succeeding $C_{k}$ s have all been produced in previous computations. Both (I) and (II) make sure that the simulation halts and does not enter an infinite loop.

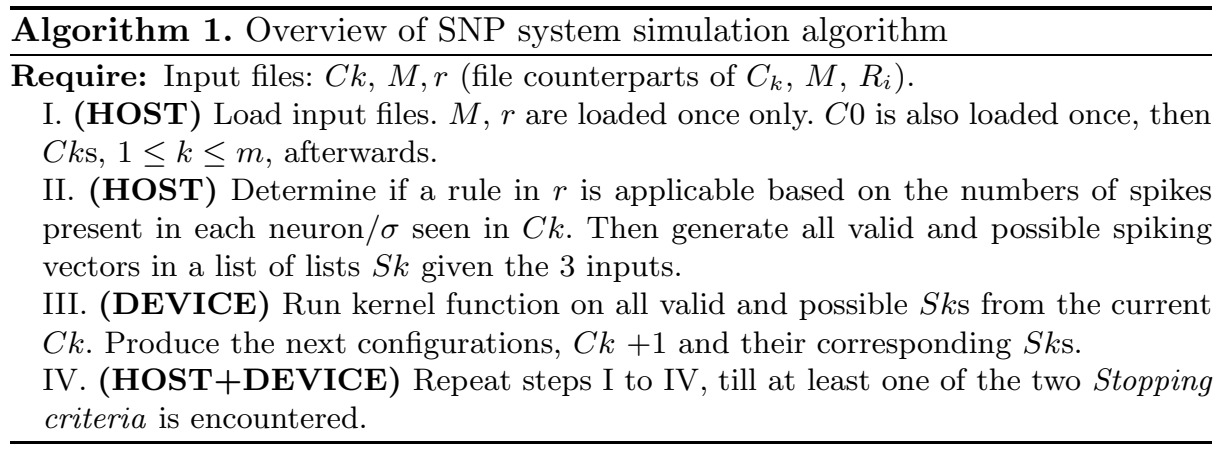


In Figure 4we see a graphical illustration of the simulation process, emphasizing the parts executed in the host/CPU and in the device/GPU. In the figure, it is assumed that there are $n$ number of $C_{k+1} \mathrm{~s}$ produced from the current $C_{k}$. The CPU executes the simulation from top to bottom, calling the kernel function, and hence the GPU, in the third box. The smaller, multiple boxes in the device/GPU part illustrate the parallel computations of all the $C_{k+1} \mathrm{~s}$ using Equation 3. Afterwards, the computed $C_{k+1} \mathrm{~s}$ are sent back to the CPU which then decides what to do next, based on Algorithm 1 part IV.

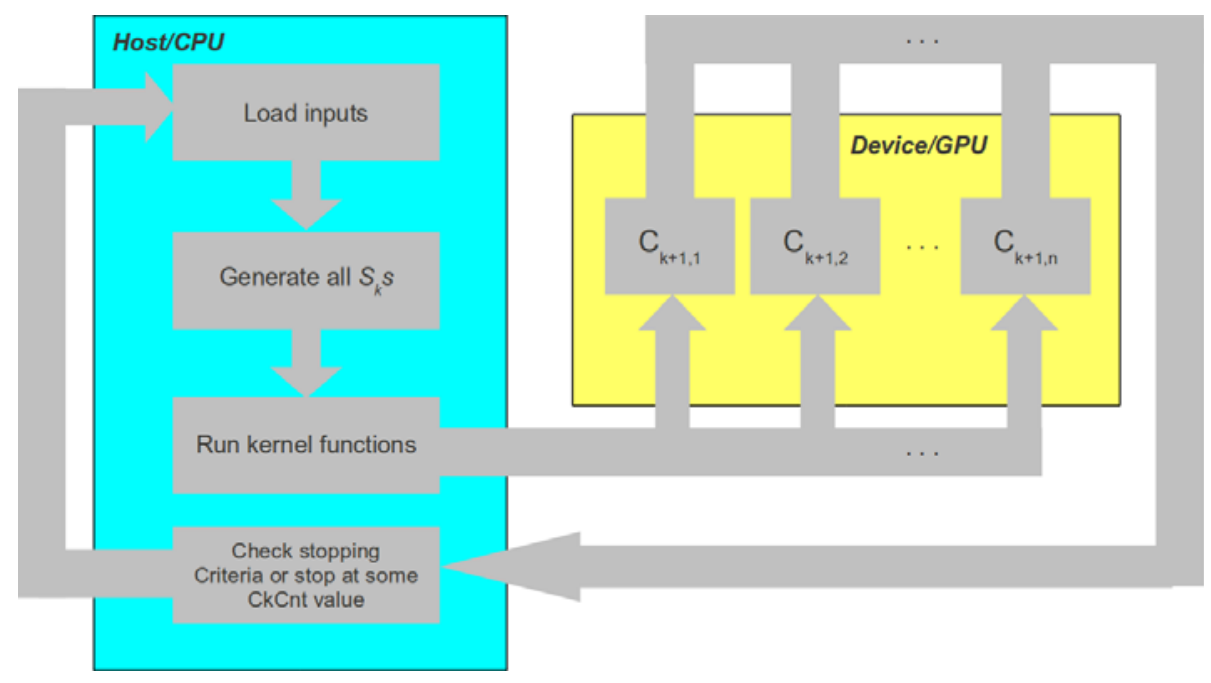

Fig. 4. Diagram showing the simulation flow, with the host and device emphasized

\section{Simulation Results and Observations}

Similar to the setup of snpgpu-sim2, snpgpu-sim3 simulated $\Pi_{1}$ and $\Pi_{2}$ using an Apple iMac running Mac OS X 10.5.8, with an Intel Core2Duo CPU with 2 cores at $2.66 \mathrm{GHz}$ (maximum clock speed) per core, and with a $6 \mathrm{MB}$ L2 cache having $4 \mathrm{~GB}$ of RAM. The GPU of the iMac is an NVIDIA GeForce 9400 graphics card at $1.15 \mathrm{GHz}$, with $256 \mathrm{MB}$ Video RAM (or around $266 \times 10^{6}$ bytes), 16 cores, running CUDA version 3.1.

In order to compare the CPU-only SNP system simulator (we designate this as snpсpu-sim) the parallel parts of snpgpu-sim3 were executed in a sequential manner. Hence, snpcpu-sim runs entirely on the CPU only, while snpgpu-sim 3 uses both the CPU and the GPU of the iMac. Both simulators use Python and C, however only snpgpu-sim3 use the CUDA enabled GPU. The simulations were done such that both snpcpu-sim and snpgpu-sim3 have the same $C_{0}$ or starting configuration as inputs. The simulations are run three times and the average of the three trial runs is taken. The simulation comparison for $\Pi_{1}$ run in both simulators is shown in Figure 5 . 


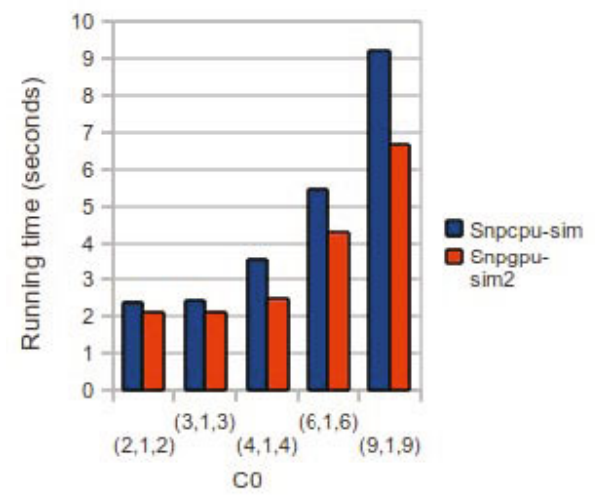

Fig. 5. Runtime graph of snpcpu-sim versus snpgpu-sim3 for $\Pi_{1}$

From Figure 5, the five $C_{0}$ values used in the simulation comparison are: $(2,1,2),(3,1,3),(4,1,4),(6,1,6)$, and $(9,1,9)$. The horizontal axis in Figure 5 are the $C_{0}$ values, while the vertical axis is the running time (in seconds) of the simulation on a given $C_{0}$. We see in Figure 5 that snpgpu-sim3 performs faster than snpcpu-sim given increasing values of $C_{0}$. A speedup of up to 1.4 times is achieved if $\Pi_{1}$ is run in snpgpu-sim3 instead of snpcpu-sim.

For $\Pi_{2}$, we introduce a variable $C k C n t$ in both simulators to limit the number of $C_{k}$ s produced to a certain integer value so the simulation will not run indefinitely. Figure 6 shows the simulation of $\Pi_{2}$ for different values of $n$ (the number of spikes in $\sigma_{1}$ of $\Pi_{2}$ is $\left.2 n\right)$ and CkCnt. In particular, the (n, CkCnt) pairs used are $(5,20),(10,25),(15,30),(20,35),(50,45),(70,50),(100,55)$, and $(200,60)$.

From Figure 6 we see that, for increasing values of $n$ and $C k C n t$, the simulation time of $\Pi_{2}$ in snpcpu-sim increases dramatically, unlike in snpgpu-sim3. The lack of a dramatic increase in simulation time in snpgpu-sim3 is because of the fact that $\Pi_{2}$ has more rules and neurons, and hence, exploits the parallel nature of the CUDA GPU all the more. A speedup of up to 6.8 times is achieved from the simulation on snpgpu-sim3 over snpcpu-sim.

We can calculate the maximum number of neurons snpgpu-sim3 can simulate using the $266 \times 10^{6}$ bytes of the NVIDIA GeForce 9400 GPU. Global memory used is $G b M e m=4 \times$ sizeof $(C k)+\operatorname{sizeof}(M)$. Here we speak in terms of the sizeof operator in the standard $\mathrm{C}$ language since we use the data type int which is of size 4 bytes or 32 bits. The length of $C k$ is the number of neurons, and $M$ is of size $R$ (rows are the number of rules) $\times C k$ (columns are number of neurons). We multiply $C k$ to 4 because we also alocate memory for $S k, C k+1$, and $M S k$ which temporarily holds the product of $M$ and $S k$, all of which are of the same size as $C k$. We therefore have the following, substituting the real value of $G b M e m$ : $266 \times 10^{6}($ bytes $)=16$ (bytes $) \times C k+4($ bytes $) \times R \times C k$. Simplifying this equation and isolating $C k$ we have $C k=266 \times 10^{6}($ bytes $) /(16($ bytes $)+4($ bytes $) \times R)$ i.e. the number of neurons that can be simulated is a function of the number of rules in the system. 


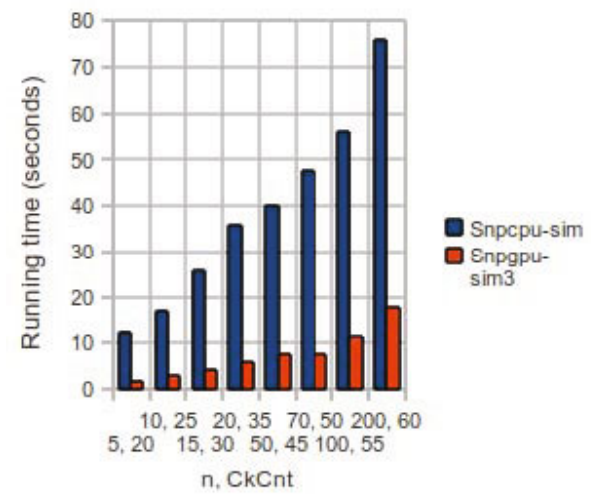

Fig. 6. Runtime graph of snpcpu-sim versus snpgpu-sim3 for $\Pi_{2}$

Additionally, aside from the iMac setup mentioned above (setup 1), a second setup was used to provide further insights of the current simulator implementation. Setup 2 consisted of two Intel Xeon E5504 processors, each having 4 cores (2 $\mathrm{GHz}$ max per core), an L2 cache of $1 \mathrm{MB}$ and an additional L3 cache of 4MB. The GPU is an NVIDIA Tesla C1060 running at $1.3 \mathrm{GHz}$ with $4 \mathrm{~GB}$ of Video RAM and having 240 cores. Setup 2 has 64bit Ubuntu 10.04.1 Linux OS, and effectively has 8 cores: $2 \times 4$ core Intel Xeon processor. The first 4 cores of processor 1 are labeled core 0 to 3 , and the next 4 cores of processor 2 are labeled core 4 to 7 .

Using the Linux command taskset, snpgpu-sim3 and snpcpu-sim were run for both $\Pi_{1}$ and $\Pi_{2}$ in setup 2 . The taskset command allows the setting of the CPU affinity of a process, thus "bonding" the process to a specified number of processor cores alone. For both snpcpu-sim and snpgpu-sim3, tests were done to set the simulation affinity to 2, 4, 8 cores, and a natural affinity setting (this is the default in the Linux OS). For simulations with 2 and 4-core affinities, the cores chosen are from the same processor i.e. either between cores 0 to 3 , or 4 to 7 , but not cores 3 and 4 together (for 2-core affinity) or 2,3,4, and 5 together (for 4-core affinity), since these core selections come from different processors and simulations will incur performance penalties. From the setup 2 tests (and for both snpсpu-sim and snpgpu-sim3), simulations done with a 2-core affinity executed in less time than with 4-core affinity simulations. Similarly, and again for both snpcpu-sim and snpgpu-sim3, 4-core affinity simulations executed in less time than 8-core affinity simulations. The 8-core affinity simulations were practically indistinguishable from the default (natural) affinity by the Linux OS.

\section{Conclusions and Future Work}

In this paper we have simulated a wider variety of SNP systems using snpgpusim3, while maintaining the efficiency of the previous simulator snpgpu-sim2. 
The extension of the previous simulator snpgpu-sim2, presented here as snpgpusim3, can now simulate larger and wider varities of SNP systems by way of more general regular expressions (those of the form (b-1)). Two SNP systems were simulated: a basic one, $\Pi_{1}$ (primarily for illustration purposes of the simulator) and a larger one (in terms of rules and neurons), $\Pi_{2}$, to exemplify the speedup when using GPUs over CPU-only simulators. The speedup of snpgpu-sim3 over snpcpu-sim for $\Pi_{1}$ went up to 1.4 times, while it was 6.8 for $\Pi_{2}$. These results show that SNP system simulation on GPUs can greatly benefit from the parallel architecture of GPUs, and that increasing the parameters (in this case neurons and rules) offer even larger speedups. This benefit in speedup is coupled with the fact that the CUDA enabled graphics cards are readily available in consumer electronic stores. These cards offer boosts in general purpose computations as co-processors of commonly used CPUs, at a fraction of the power consumption of CPU clusters.

From setup 2 which is a "larger" setup compared to setup 1, we see that for "small" systems e.g. $\Pi_{1}$, the CPU can outperform the GPU for snpgpu-sim3. Setup 2 helped validate and support the performance results from setup 1 using snpgpu-sim3. The current simulation in Algorithm 1 is thus recommended for up to 4 cores on a single processor only, as the CPU part is not multi-threaded (only the GPU part is). Using more than 4 cores on a single processor, or more than one core from more than one processor in a multiprocessor setup will not yield better performance. Alternatively, simulating "larger" SNP systems can capitalize on the massively parallel architecture of GPUs compared to multi-core CPUs, and thus increase the speedup even further.

For future work, a generic $\mathrm{P}$ system parser based using P-lingua would be able to provide a more standardized input parser and formatting for the GPU based SNP system simulator. P-lingua is programming environment for Membrane computing which takes in as input a certain $\mathrm{P}$ system variant, and outputs a standardized XML file format for the simulator to use. This standardized simulator input format would then be carried on to other simualtors when simulating other $\mathrm{P}$ system variants.

Further understanding of the CUDA architecture as well as adapting the simulator to GPUs with more cores and their newer parallel technologies, as well as their subtleties, are also for future work. Increasing the level of simulation parallelism is also part of this work, to gain even higher performance speedups over CPU only simulations.

More SNP system variants can be simulated by extending the current GPU simulator (with neuron budding, with delays et al). Lastly, the use of the simulator to empirically test the models of problems solved with SNP systems is also a future work.

Acknowledgments. Francis George Cabarle is supported by the DOST-ERDT program. Henry Adorna is funded by the DOST-ERDT research grant and the Alexan professorial chair of the UP Diliman Department of Computer Science. 
Miguel A. Martínez-del-Amor is supported by "Proyecto de Excelencia con Investigador de Reconocida Valía" of the "Junta de Andalucía" under grant P08TIC04200, and by the project TIN2009-13192 of the "Ministerio de Ciencia e Innovación" of Spain, both co-financed by FEDER funds.

\section{References}

1. Cabarle, F., Adorna, H., Martínez-del-Amor, M.A.: Simulating Spiking Neural P systems without delays using GPUs. In: Proceedings of the 9th Brainstorming Week on Membrane Computing, Sevilla, Spain (February 2011)

2. Cabarle, F., Adorna, H., Martínez-del-Amor, M.A.: An Improved GPU Simulator For Spiking Neural P Systems. In: IEEE Sixth International Conference on Bio-Inspired Computing: Theories and Applications, Penang, Malaysia (September 2011), doi:10.1109/BIC-TA.2011.37

3. Cecilia, J.M., García, J.M., Guerrero, G.D., Martínez-del-Amor, M.A., PérezHurtado, I., Pérez-Jiménez, M.J.: Simulation of P systems with active membranes on CUDA. Briefings in Bioinformatics 11(3), 313-322 (2010)

4. Cecilia, J.M., García, J.M., Guerrero, G.D., Martínez-del-Amor, M.A., PérezHurtado, I., Pérez-Jiménez, M.J.: Simulating a P system based efficient solution to SAT by using GPUs. Journal of Logic and Algebraic Programming 79(6), 317-325 (2010)

5. Chen, H., Ionescu, M., Ishdorj, T.-O., Păun, A., Păun, G., Pérez-Jiménez, M.: Spiking neural P systems with extended rules: universality and languages. Natural Computing: an International Journal 7(2), 147-166 (2008)

6. Ciobanu, G., Wenyuan, G.: P Systems Running on a Cluster of Computers. In: Martín-Vide, C., Mauri, G., Păun, G., Rozenberg, G., Salomaa, A. (eds.) WMC 2003. LNCS, vol. 2933, pp. 123-139. Springer, Heidelberg (2004)

7. Díaz, D., Graciani, C., Gutiérrez, M.A., Pérez-Hurtado, I., Pérez-Jiménez, M.J.: Software for P systems. In: Păun, G., Rozenberg, G., Salomaa, A. (eds.) The Oxford Handbook of Membrane Computing, ch. 17, pp. 437-454. Oxford University Press, Oxford (2009)

8. Fatahalian, K., Sugerman, J., Hanrahan, P.: Understanding the efficiency of GPU algorithms for matrix-matrix multiplication. In: Proceedings of the ACM SIGGRAPH/EUROGRAPHICS Conference on Graphics Hardware (HWWS 2004), pp. 133-137. ACM, NY (2004)

9. Garland, M., Kirk, D.B.: Understanding throughput-oriented architectures. Communications of the ACM 53(11), 58-66 (2010)

10. Harris, M.: Mapping computational concepts to GPUs. In: ACM SIGGRAPH 2005 Courses, NY, USA (2005)

11. Ionescu, M., Păun, G., Yokomori, T.: Spiking Neural P Systems. Journal Fundamenta Informaticae 71(2,3), 279-308 (2006)

12. Kirk, D., Hwu, W.: Programming Massively Parallel Processors: A Hands on Approach, 1st edn. Morgan Kaufmann, MA, USA (2010)

13. Klöckner, A., Pinto, N., Lee, Y., Catanzaro, B., Ivanov, P., Fasih, A.: PyCUDA: GPU Run-Time Code Generation for High-Performance Computing, no. 2009-40. Scientific Computing Group, Brown University, RI, USA (2009)

14. Păun, G., Ciobanu, G., Pérez-Jiménez, M. (eds.): Applications of Membrane Computing. Natural Computing Series. Springer, Heidelberg (2006) 
15. Nguyen, V., Kearney, D., Gioiosa, G.: A Region-Oriented Hardware Implementation for Membrane Computing Applications. In: Păun, G., Pérez-Jiménez, M.J., Riscos-Núñez, A., Rozenberg, G., Salomaa, A. (eds.) WMC 2009. LNCS, vol. 5957, pp. 385-409. Springer, Heidelberg (2010)

16. Zeng, X., Adorna, H., Martínez-del-Amor, M.Á., Pan, L., Pérez-Jiménez, M.J.: Matrix Representation of Spiking Neural P Systems. In: Gheorghe, M., Hinze, T., Păun, G., Rozenberg, G., Salomaa, A. (eds.) CMC 2010. LNCS, vol. 6501, pp. 377-391. Springer, Heidelberg (2010)

17. NVIDIA corporation, NVIDIA CUDA C programming guide, version 3.0. NVIDIA, CA, USA (2010) 